\title{
OTUD7B stabilizes estrogen receptor $a$ and promotes breast cancer cell proliferation
}

\author{
Jianing Tang ${ }^{1}$, Zeyu Wu', Zelin Tian', Wei Chen ${ }^{1}$ and Gaosong Wu ${ }^{1}$
}

\begin{abstract}
Breast cancer is the most common malignancy in women worldwide. Estrogen receptor a (ERa) is expressed in 70\% of breast cancer cases and promotes estrogen-dependent cancer progression. In the present study, we identified OTU domain-containing 7B (OTUD7B), a deubiquitylase belonging to A20 subgroup of ovarian tumor protein superfamily, as a bona fide deubiquitylase of ERa in breast cancer. OTUD7B expression was found to be positively correlated with ERa in breast cancer and associated with poor prognosis. OTUD7B could interact with, deubiquitylate, and stabilize ERa in a deubiquitylation activity-dependent manner. Depletion of OTUD7B decreased ERa protein level, the expression of ERa target genes, and the activity of estrogen response element in breast cancer cells. In addition, OTUD7B depletion significantly decreased ERa-positive breast cancer cell proliferation and migration. Finally, overexpression of ERa could rescue the suppressive effect induced by OTUD7B depletion, suggesting that the ERa status was essential to the function of OTUD7B in breast carcinogenesis. In conclusion, our study revealed an interesting post-translational mechanism between ERa and OTUD7B in ERa-positive breast cancer. Targeting the OTUD7B-ERa complex may prove to be a potential approach to treat patients with ERa-positive breast cancer.
\end{abstract}

\section{Introduction}

Breast cancer ranks the most prevalent cancer and the second leading cause of cancer-related death in women worldwide ${ }^{1}$. Based on the expression of estrogen receptor (ER), progesterone receptor (PR), and human epidermal growth factor receptor 2 (HER-2), breast cancer can be divided into at least three subtypes: luminal, HER2-enriched, and triple-negative, which exhibit different histopathological features and treatment sensitivities ${ }^{2}$.

Approximately $70 \%$ breast cancer cases are positive for estrogen receptor alpha $(E R \alpha)$, which is known as the biomarker and one of the most successful molecular targets for endocrine therapy $y^{3,4}$. It is structurally organized into several domains: transactivation domains AF-1 and AF-2, which recruit both transcriptional coactivators and corepressors; the DNA-binding domain (DBD), which is required for the

\footnotetext{
Correspondence: Gaosong Wu (wugaosongtj@163.com)

'Department of Thyroid and Breast Surgery, Zhongnan Hospital of Wuhan

University, Wuhan, China

These authors contributed equally: Jianing Tang, Zeyu Wu

Edited by A. Peschiaroli
}

specific binding to estrogen response elements (ERE) in enhancers or promoters, and the ligand-binding domain (LBD), which is recognized by the 17 beta estradiol hormone $(\mathrm{E} 2)^{5,6}$. ER $\alpha$ plays a central role in the signal transduction pathways of breast cancer cells, and upregulation of ER $\alpha$ is associated with the initiation and progression of breast cancer $^{7,8}$. The activity of ER $\alpha$ is essential for cell cycle progression in that it accelerates the G1-S-phase transition. Overexpression of ER $\alpha$ promotes breast cancer cell growth by increasing the expression level of oncogenic proteins, including cyclin D1 and c-myc ${ }^{9}$. Since ER $\alpha$ and its signaling pathways are essential in the development of breast cancer cells, detection of ER $\alpha$ is considered as an important indicator for implementation of endocrine therapy ${ }^{10}$. Despite patients with $E R \alpha$-positive tumors having high sensitivity to endocrine therapy, $30-50 \%$ of them will suffer from later relapse and develop acquired resistance. Distant metastases and resistance to therapy are the major causes of death in breast cancer patients ${ }^{11-13}$. Acquired resistance to endocrine therapy is a common problem in ER $\alpha$-positive breast cancer, while the mechanisms underlying this resistance are not 


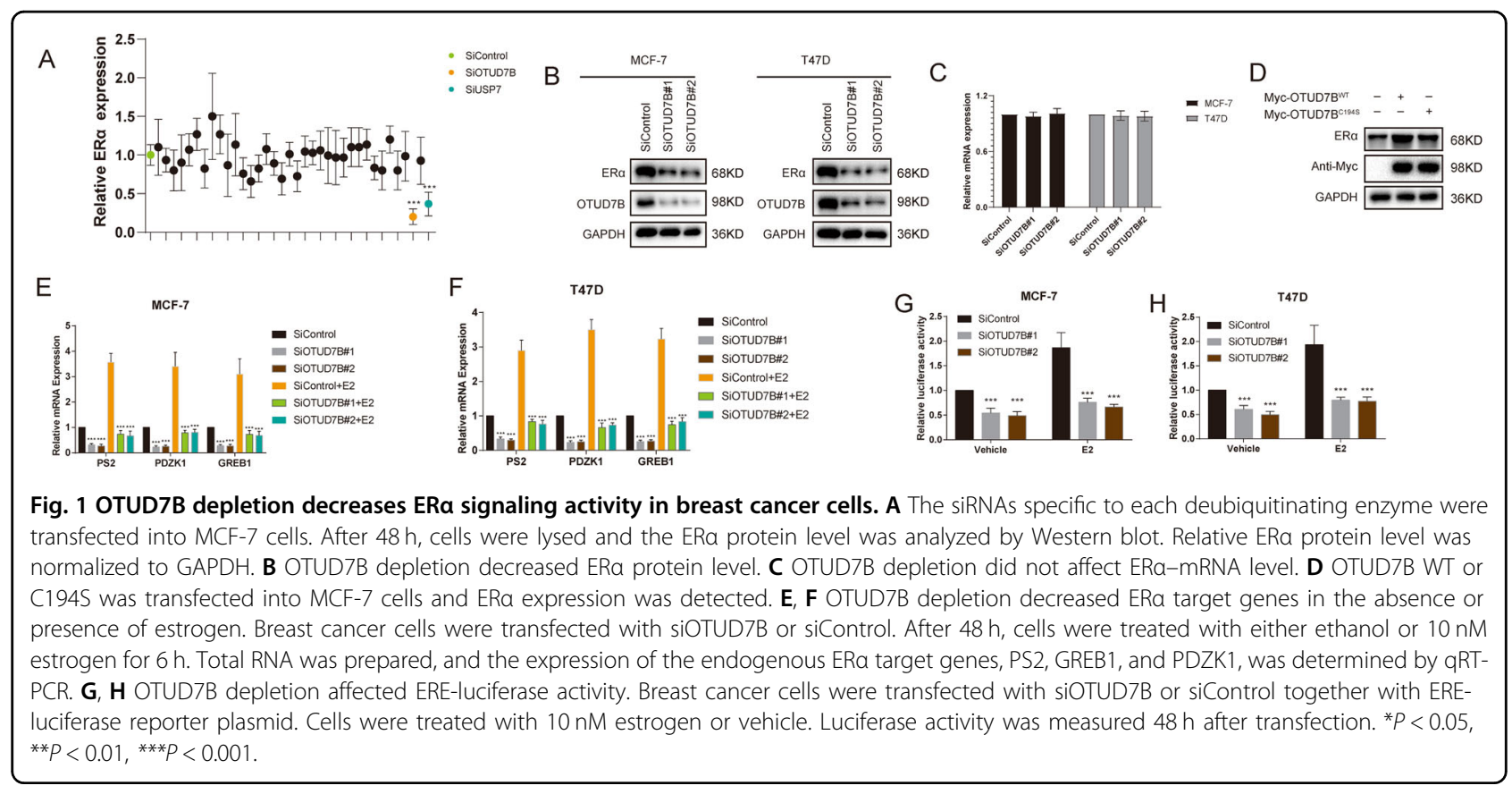

completely defined $^{14-17}$. Thus, it is of utmost importance to understand the dysregulation of ER $\alpha$ signaling.

ER $\alpha$ can be modified by several kinds of posttranslational modifications such as ubiquitination, SUMOlyation, and phosphorylation ${ }^{18-20}$. Recent studies have indicated that many proteins and mechanisms are involved in the regulation of ER $\alpha$ stability via the ubiquitin-proteasome system (UPS). Certain E3-ubiquitin ligases, including BRCA1, BARD1, E6AP, CHIP, MDM2, and SKP2, can increase the polyubiquitination to ER $\alpha$ lysine residues and induce its degradation by the $26 \mathrm{~S}$ proteasome $\mathrm{e}^{21-26}$. Several E3 ligases are demonstrated to facilitate $E R \alpha$ signaling via stabilizing $E R \alpha$ protein. RNF31, SHAPRIN, TRIM11, and RNF8 enhance ER $\alpha$ stability by inducing its monoubiquitination ${ }^{27-30}$. TRIM56 stabilizes ER $\alpha$ targeting via promoting K63linked ubiquitination on ER $\alpha$ protein ${ }^{31}$. In addition to E3ubiquitin ligases, deubiquitinating enzymes (DUB) also modulate the stability of $E R \alpha$ protein in breast cancer, which functions to cleave ubiquitin chains from ER $\alpha$ proteins to modulate its degradation. A previous study demonstrated that USP7 is a DUB of ER $\alpha$ and promoted breast cancer progression ${ }^{32}$. However, the DUB responsible for $E R \alpha$ deubiquitination is largely unknown.

In the present study, we screened a DUB siRNA library and found that OTUD7B was a possible deubiquitinase responsible for $E R \alpha$ deubiquitination and stabilization in ER $\alpha$-positive cancer. We also found that OTUD7B promoted cell proliferation and migration through ER $\alpha$. Overall, our study has demonstrated that OTUD7B is a novel deubiquitinating enzyme of ER $\alpha$ and may prove to be a potential target for breast cancer intervention.

\section{Results}

OTUD7B depletion inhibits ERa signaling pathway activity

We initially utilized a siRNA screen library to identify the deubiquitinating enzymes responsible for ER $\alpha$ deubiquitination and stabilization in ER $\alpha$-positive breast cancer. Four nonoverlapping siRNA mixtures specific for each of the DUBs were transfected into MCF-7 cells; it was found that silencing OTUD7B markedly decreased ER $\alpha$ (Fig. 1A). We then depleted OTUD7B with two nonoverlapping siRNAs separately in MCF-7 and T47D cells to further validate the function of OTUD7B in regulating ER $\alpha$; as shown in Fig. 1B, OTUD7B depletion significantly decreased the ER $\alpha$ protein levels. Consistently, ectopic expression of OTUD7B profoundly upregulated ER $\alpha$. While the catalytically inactive mutant C194S (OTUD7B C194S) lost its ability to upregulate $E R \alpha$, suggesting that OTUD7B regulated ER $\alpha$ in a DUBactivity-dependent manner (Fig. 1C). We examined the expression of ER $\alpha$ target genes (PS2, PDZK1, and GREB1) and found that depletion of OTUD7B dramatically decreased the transcripts of PS2, PDZK1, and GREB1 in both estrogen and vehicle conditions (Fig. 1D, E). In addition, we measured the ER $\alpha$-luciferase reporter gene activity by OTUD7B depletion to determine whether OTUD7B depletion affected the ER $\alpha$ transcriptional activity It was found that depletion of OTUD7B decreased the ER $\alpha$-luciferase reporter gene activity in the presence or absence of estrogen (Fig. 1F, G). Overexpression of WT OTUD7B in MCF-7 cells increased the transcripts of ER $\alpha$ target genes and the ER $\alpha$-luciferase reporter gene activity (Fig. S1). All these results demonstrated that OTUD7B was a regulator of the ER $\alpha$ signaling pathway. 


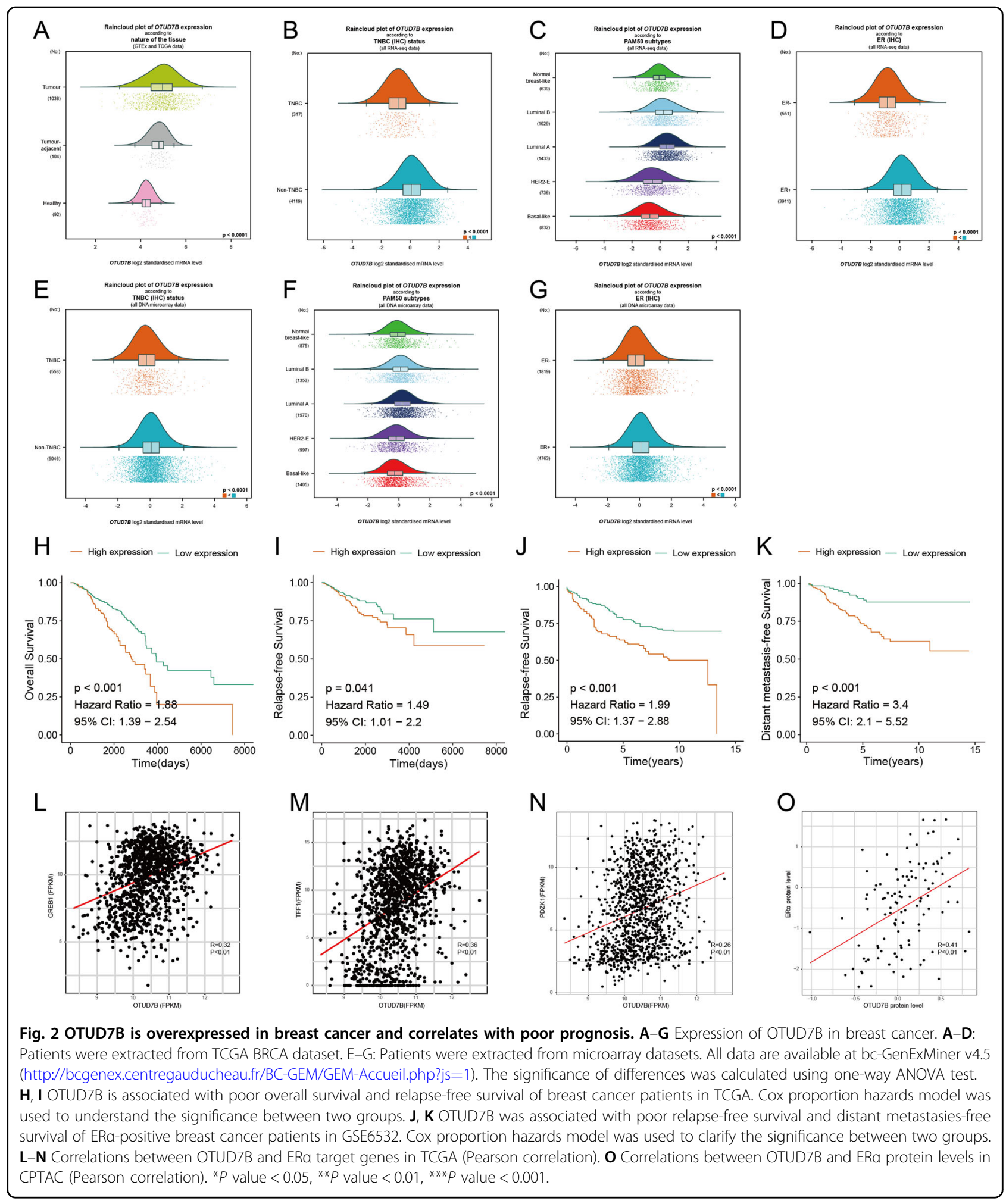

OTUD7B is associated with ERa protein levels in human breast cancer samples and poor prognosis

OTUD7B expression was analyzed in breast cancers using bc-GenExMiner v4.5 (http://bcgenex.centregauducheau.fr/
BC-GEM/GEM-Accueil.php?js=1), a statistical mining tool of published annotated breast cancer transcriptomic data (DNA microarrays $[n=10716]$ and RNA-seq $[n=4716]$ ). As shown in Fig. 2, OTUD7B was highly expressed in breast 
cancer samples, especially in the luminal A subtype (Fig. 2A-G). Then we performed survival analysis of OTUD7B based on TCGA, and found that OTUD7B expression was a poor prognostic factor for breast cancer patients (Fig. 2H, I). As OTUD7B was upregulated in $E R \alpha$-positive breast cancer patients and associated with the ER $\alpha$ protein level, we then analyzed its prognostic value in ER $\alpha$-positive breast cancer from GES6532, and observed that high expression of OTUD7B was associated with poor prognosis of patients with ER $\alpha$-positive breast cancer (Fig. 2J, K). We further analyzed the correlation between OTUD7B and ER $\alpha$ target gene expressions. From TCGA database, we observed that OTUD7B was positively correlated with PS2, PDZK1, and GREB1 expression (Fig. 2L-N). Based on the global proteome data of 105 TCGA breast cancer samples from the Clinical Proteomic Tumor Analysis Consortium (https://cptac-data-portal.georgetown.edu/cptacPublic/), we also observed a positive correlation between OTUD7B and ER $\alpha$ protein levels (Fig. 2O). Consistently, immunohistochemistry (IHC) analysis of two tissue microarrays (TMA) indicated a positive correlation between OTUD7B and ER $\alpha$ staining, and high expression of OTUD7B indicated a poor prognosis (Fig. 3). Further analysis demonstrated that OTUD7B expression was correlated with the ER $\alpha$ status, the lymph-node metastasis status, and tumor size.

\section{OTUD7B interacts with ERa}

The results of immunostaining demonstrated that ER $\alpha$ and OTUD7B colocalized both in the nucleus and cytosol of breast cancer cells (Fig. 4A). We found that endogenous OTUD7B coimmunoprecipitated with endogenous $\mathrm{ER} \alpha$ in the co-immunoprecipitation (Co-IP) experiment (Fig. 4B). GST pull-down assay showed that OTUD7B directly interacted with ER $\alpha$ in vitro (Fig. 4C). Additionally, deletion analysis demonstrated that OTUD7B physically interacted with the AF1 domain of ER $\alpha$ (Fig. 4D, E).

\section{OTUD7B deubiquitylates ERa}

The interaction between OTUD7B and ER $\alpha$ suggested that ER $\alpha$ might be a substrate of OTUD7B, and therefore we evaluated the possibility of ER $\alpha$ deubiquitylation by OTUD7B. It was found that OTUD7B deletion dramatically decreased ER $\alpha$ protein level, and this effect could be reversed by addition of the proteasome inhibitor MG132 or overexpression of OTUD7B-WT, but not its catalytically inactive mutant OTUD7B ${ }^{\mathrm{C} 194 \mathrm{~S}}$ (Fig. 5A, B). We then treated cells with the protein synthesis inhibitor cycloheximide to prove that OTUD7B affected ER $\alpha$ stability. The stability of $E R \alpha$ was significantly decreased in cells depleted of OTUD7B (Fig. 5C). In cells overexpressing OTUD7B-WT, but not OTUD7B ${ }^{\mathrm{C} 194 \mathrm{~S}}$, the half-life of ER $\alpha$ was prolonged (Fig. 5D). Depletion of OTUD7B significantly increased the level of ubiquitinated ER $\alpha$ in MCF-7 cells. We also observed
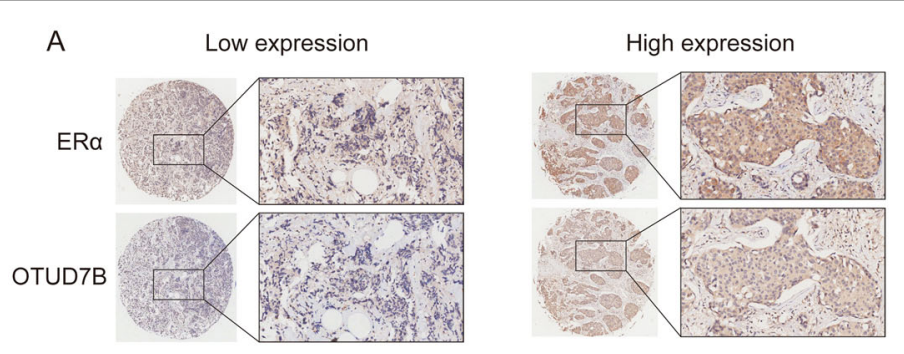

B
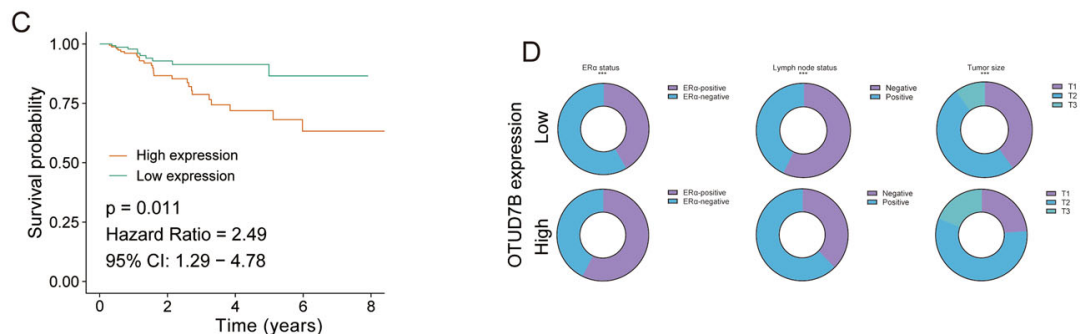

Fig. 3 OTUD7B correlates with ERa protein levels and poor prognosis in human breast cancer samples. Tissue microarray (HBreD140Su04) was obtained from Shanghai Biochip Company Co., Ltd, Shanghai, China. The tissue microarray contained 140 breast cancer specimens. A The typical staining of ERa and OTUD7B in breast cancer specimens. Specific primary antibodies against ERa (Proteintech, China) and OTUD7B (Proteintech, China) were used for IHC. B ERa positively correlated with OTUD7B in breast cancer samples (Pearson correlation). C High expression of OTUD7B was associated with poor prognosis. Cox proportion hazards model was used to understand the significance between two groups. D OTUD7B expression was associated with the ERa status, lymph-node status, and tumor size. The characteristics were compared between OTUD7B low-/high-groups using chi-square or Fisher's exact tests. ${ }^{*} P$ value $<0.05$, ${ }^{* *} P$ value $<0.01$, ${ }^{* *} P$ value $<0.001$. 

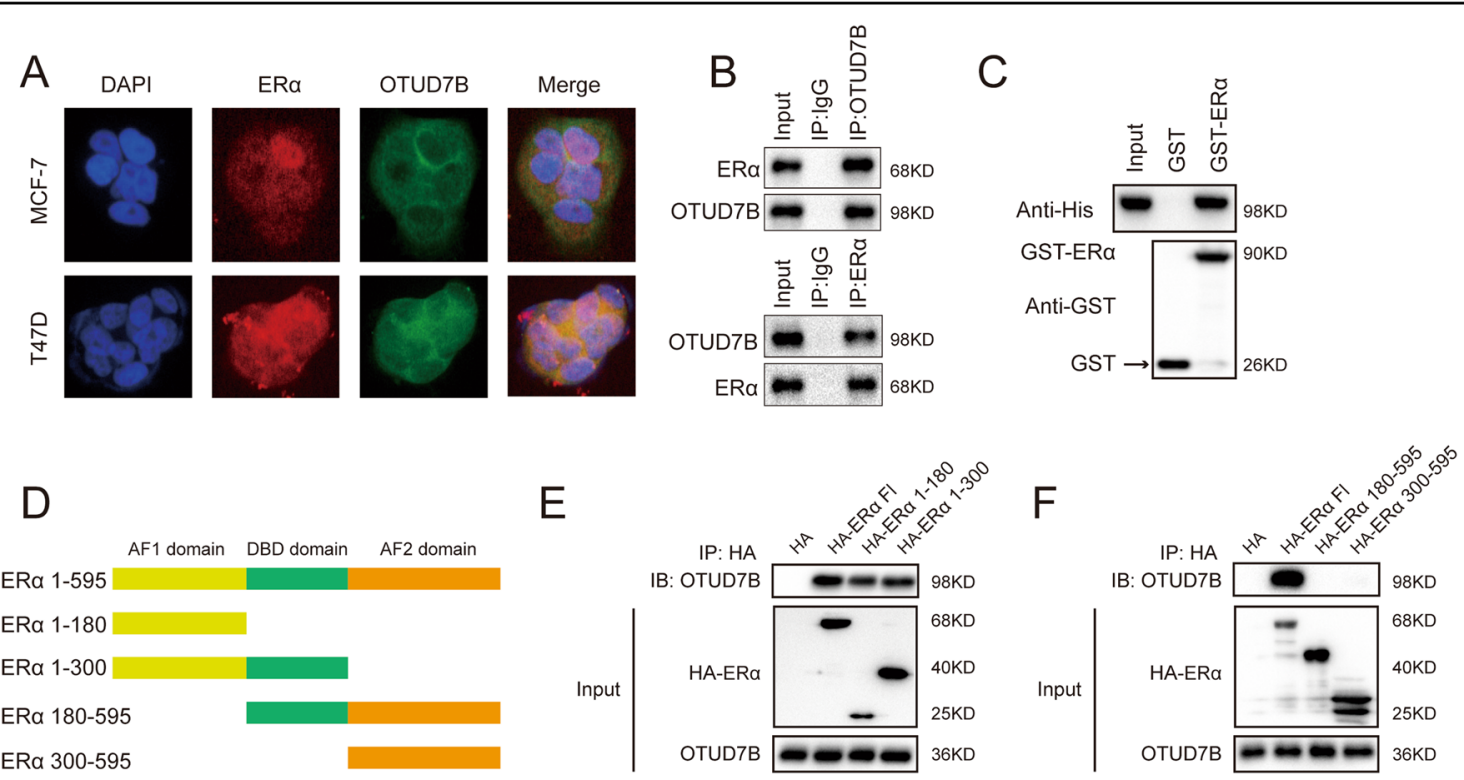

Fig. 4 OTUD7B associates with ERa. A An immunofluorescence assay demonstrated that OTUD7B and ERa at least partially colocalized in MCF7 and T47D cells. B Co-IP assay revealed an association between endogenous OTUD7B and ERa in MCF-7 cells. MCF-7 cells were harvested with RIPA lysis buffer. Co-IP was performed using antibody as indicated. C Purified His-OTUD7B was incubated with GST- ERa or GST protein. The interacted OTUD7B was detected via western blot. D ER alpha domain structure and deletion mutants used in the study. E, F OTUD7B interacted with ERa through its AF1 domain. HEK293 cells were transfected with $2 \mu \mathrm{g}$ Myc-OTUD7B together with HA- ERa full length or mutants. After $24 \mathrm{~h}$, cells were harvested with NP-40 lysis buffer. Co-IP was performed using Myc antibody. The possible interacted ERa domains were detected by HA antibody.

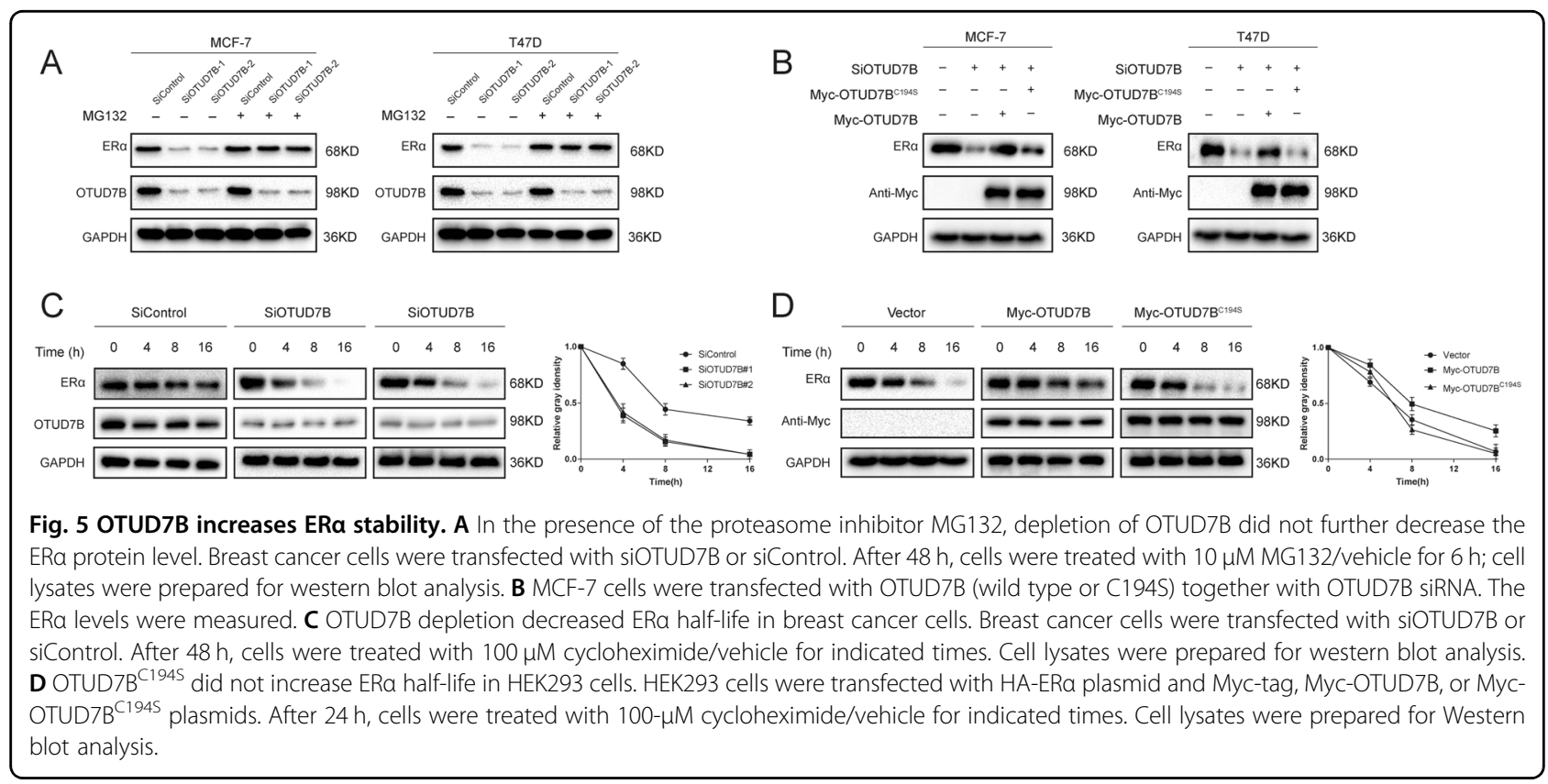

that USP7 depletion induced ER $\alpha$ ubiquitination (Fig. 6A, B). Conversely, ectopic expression of OTUD7B-WT, but not OTUD7B ${ }^{\mathrm{C} 194 \mathrm{~S}}$, markedly decreased ER $\alpha$ ubiquitylation in cells both in vivo and in vitro (Fig. $6 \mathrm{C}$ and Fig. S2). In vivo deubiquitylation assays showed that OTUD7B directly removed the ubiquitin chain of ER $\alpha$ in a time- and dose- dependent manner (Fig. 6D, E). Furthermore, OTUD7B decreased the ER $\alpha$ ubiquitylation induced by the E3 ligase TRIM8 (Fig. 6F) ${ }^{33}$. We further performed ubiquitination assay with a series of ubiquitin mutants to investigate which type of ubiquitin chain of ER $\alpha$ was deubiquitylated by OTUD7B. It was found that OTUD7B efficiently removed 


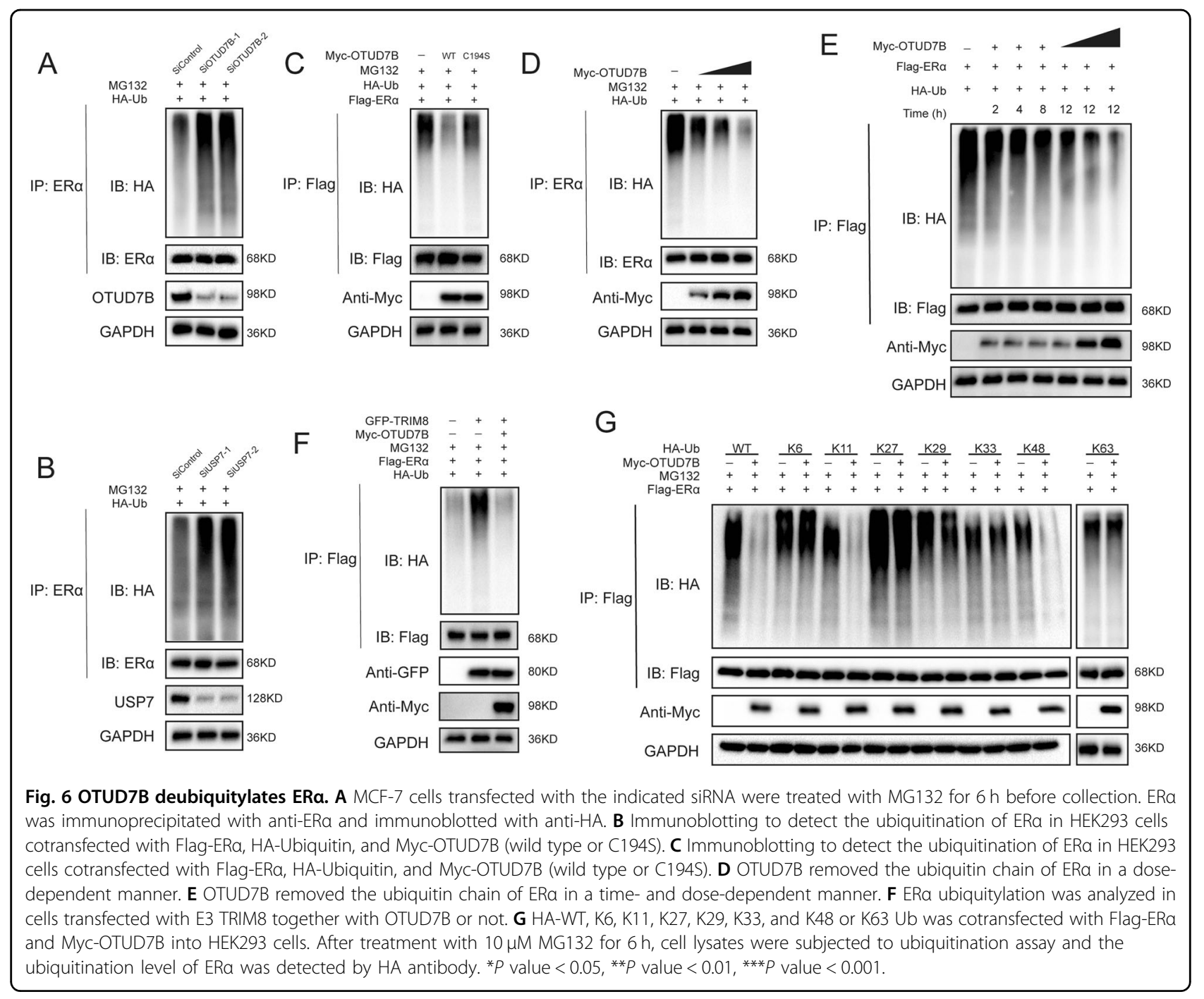

the K11- and K48-linked ubiquitin chain on ER $\alpha$ (Fig. 6F). Taken together, OTUD7B was identified as a specific DUB, which depolyubiquitylated and stabilized ER $\alpha$.

\section{OTUD7B regulates cell proliferation and migration through ERa}

We next examined the role of OTUD7B in regulating breast cancer progression. Our results demonstrated that depletion of OTUD7B significantly decreased cell proliferation and increased the population in G1 phases, indicating that OTUD7B may regulate G1-to-S transition in $\mathrm{ER} \alpha$-positive breast cancer cells (Fig. 7A, B). The results of clone formation assay revealed that OTUD7B depletion dramatically decreased the clone formation capability in MCF-7 and T47D cells (Fig. 7C). Consistently, EdU incorporation assay indicated that DNA synthesis was inhibited in MCF-7 and T47D cells treated with OTUD7B siRNAs (Fig. 7D, E). Furthermore, depletion of OTUD7B significantly decreased cell migration capacity as revealed by wound-healing assay (Fig. 7F). We then expanded the experiments using stable knockdown cell lines, and USP7 was used as the positive control. As shown in Fig. S3, stable knockdown of OTUD7B or USP7 markedly inhibited breast cancer cell proliferation and migration, reduced tumor growth and metastasis in vivo (Fig. S3). To determine the mechanism of OTUD7B in regulating breast cancer cell proliferation and migration by stabilizing $E R \alpha$, we performed rescue experiments by ectopicexpressing ER $\alpha$ in OTUD7B- knockdown MCF-7 cells. CCK8 assay indicated that overexpression of ER $\alpha$ largely recovered the proliferation ability of MCF-7 cells (Fig. 8A). Increased ER $\alpha$ expression reversed the clone formation ability of MCF-7 cells (Fig. 8B). Consistently, $\mathrm{ER} \alpha$ overexpression also facilitated the DNA synthesis in MCF-7 cells depleted with OTUD7B (Fig. 8C). Woundhealing assay showed that the suppressive function induced by OTUD7B depletion was largely reversed by ER $\alpha$ overexpression (Fig. 8D). Knockdown of OTUD7B 


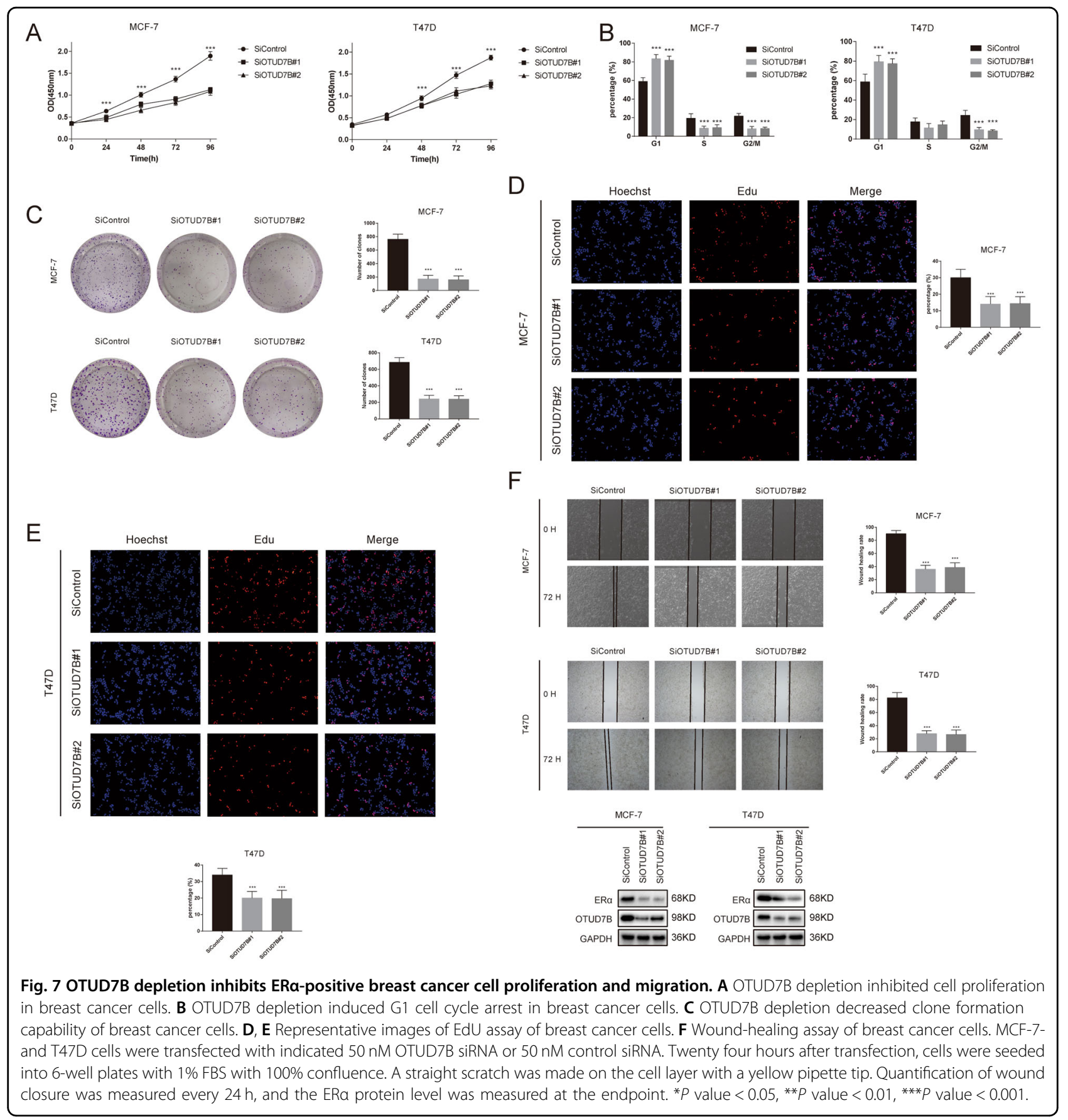

significantly inhibited tumor growth in vivo, while the restoration of ER $\alpha$ expression abolished the inhibition induced by OTUD7B depletion (Fig. 8E). In addition, depletion of OTUD7B by using in vivo-optimized RNAi also significantly reduced tumor growth (Fig. 8F). To further confirm that ER $\alpha$ is required for OTUD7B to promote breast cancer cell proliferation and migration, we overexpressed OTUD7B in ER $\alpha$-depleted cells. We found that OTUD7B could not promote the proliferation and migration of MCF-7 cells depleted with ER $\alpha$ (Fig. S4).
These results indicated that OTUD7B promoted breast cancer cell proliferation and migration, at least partially, via the regulation of ER $\alpha$.

\section{Discussion}

Breast cancer is the most common cancer in women worldwide. Seventy percent of cases are ER $\alpha$ positive ${ }^{34}$. $E R \alpha$ belongs to the nuclear receptor superfamily of transcriptional factors, which was originally cloned from MCF7 cell in $1985^{35}$. Targeting ER $\alpha$ has been demonstrated to 


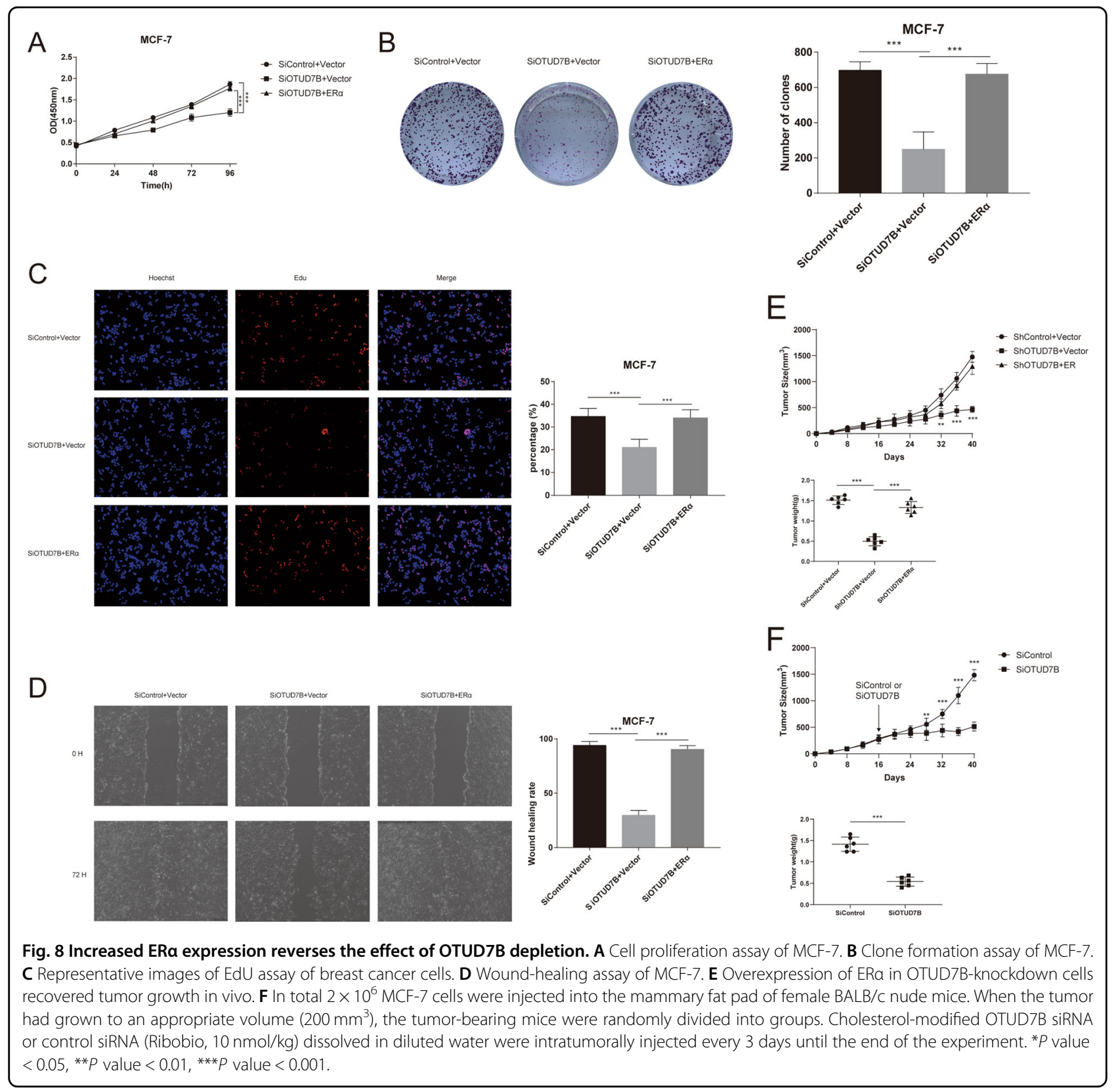

be one of the most successful strategies for endocrine therapy in ER $\alpha$-positive breast cancer patients because of its sensitivity and effectiveness ${ }^{36}$. However, endocrine resistance remains an important concern in breast cancer therapy, and several confirmed and hypothetical mechanisms of endocrine resistance have been reported. Singlestrand conformation polymorphism (SSCP) reveals a 42base-pair replacement in exon 6 of the estrogen receptor complementary DNA of a tamoxifen-resistant tumor ${ }^{37}$. In addition to $\mathrm{ER} \alpha$ gene mutations in $\mathrm{AF}-2$ domain, breast cancer cells can get endocrine resistance through several kinds of ER $\alpha$ modifications, such as phosphorylation, acetylation, and ubiquitination. P300 could directly acetylate the ER $\alpha$ at lysine residues within the ER $\alpha$ hinge/ ligand-binding domain and subsequently promote the ERsignaling activity. Substitution of these residues with charged or polar residues can enhance ER $\alpha$ hormone sensitivity $^{38}$. ER $\alpha$ phosphorylation at certain sites can have a profound impact on ER $\alpha$ function in breast cancer. For example, the phosphorylation at Y537 site changes helix loop conformation and then enhances ligand binding/ coactivator-binding efficiency ${ }^{39,40}$. Accumulating evidence has indicated that ER signaling and turnover is tightly linked to the ubiquitin-proteasome system. However, studies exploring the DUB responsible for ER $\alpha$ are limited. In this study, we identified that OTUD7B, which was highly 
expressed in human breast cancer samples, was a novel $E R \alpha$ co-regulator through post-translational modification. OTUD7B was associated with ER $\alpha$ and inhibits ER $\alpha$ polyubiquitination and degradation. In particular, OTUD7B is essential in breast carcinogenesis.

OTUD7B, a DUB belonging to A20 subgroup of ovarian tumor (OTU) protein superfamily, was previously described as a cell cycle-regulated DUB. OTUD7B can remove K11-linked ubiquitin chains from APC/C substrates at the exit of mitosis by binding, deubiquitinating, and stabilizing the anaphase-promoting complex/cyclosome (APC) substrates, thus contributing to mitotic progression and proliferation ${ }^{41}$. OTUD7B has a preference to remove K11linked ubiquitin chains from substrates, other studies also suggested that OTUD7B deubiquitinated K48- and K63linked ubiquitin chains ${ }^{42-44}$. OTUD7B negatively regulated noncanonical NF- $\mathrm{kB}$ pathway by deubiquitinating K48-linked polyubiquitin chains of TRAF3, an inhibitor of the NF-kappa-B pathway, thereby preventing TRAF3 proteolysis and overactivation of noncanonical NF- $k \mathrm{~B}^{45}$. OTUD7B also enhanced EGF-induced growth and migration signals by mediating the deubiquitination of $\mathrm{EGFR}^{46}$. Interestingly, OTUD7B regulated the HIF transcriptional activity via directly affecting HIF-1a protein degradation in a proteasome-independent way, possibly through chaperone-mediated autophagy ${ }^{47}$. Besides, OTUD7B had been demonstrated to be able to stabilize Sox2 and maintain neural progenitor cell property through the removal of ubiquitin from Sox $2^{48}$. Although previous evidence has shown that OTUD7B plays a crucial role in carcinogenesis, the molecular mechanisms of OTUD7B participation in the progression of breast cancer remain elusive.

OTUD7B is overexpressed in the breast cancer tissue as compared with that in the paired adjacent normal tissue, and high expression of OTUD7B is associated with poor prognosis of breast cancer patients ${ }^{46}$. In the present study, we first found that OTUD7B was a novel modulator in controlling ER $\alpha$ ubiquitination and stability, which depends on its DUB activity. Depletion of OTUD7B significantly decreased the ER $\alpha$ protein level and inhibited the ER $\alpha$ signaling activity. Based on the analysis of public data available in bc-GenExMiner, we found that OTUD7B was highly expressed in breast cancer tissue, especially in the ER $\alpha$-positive subtype, and that high expression of OTUD7B was associated with poor prognosis. Consistently, we observed an intimate correlation between OTUD7B expression and ER $\alpha$ protein level according to tissue microarray staining from 140 human breast cancer samples. In addition, survival analysis indicated that OTUD7B expression is associated with poor clinical outcomes. We further explored the molecular mechanism of OTUD7B in regulating ER $\alpha$, and found that ER $\alpha$ protein level was significantly decreased upon OTUD7B depletion.
In addition, ectopic expression of OTUD7B profoundly upregulated ER $\alpha$ in a dose-dependent manner. While the catalytically inactive mutant lost its ability to upregulate $\mathrm{ER} \alpha$. When cells were treated with the CHX, the half-life of $E R \alpha$ was significantly shortened in cells depleted of OTUD7B, but prolonged in cells overexpressing OTUD7B. We then tested whether ubiquitin-proteasome system (UPS) was required to $\mathrm{ER} \alpha$ degradation induced by OTUD7B depletion, and found that MG132 largely recovered the decreased ER $\alpha$ expression induced by OTUD7B silence. We also identified that OTUD7B colocated with ER $\alpha$. The Co-IP experiment demonstrated that endogenous OTUD7B coimmunoprecipitated with endogenous ER $\alpha$. GST pull-down assay showed that OTUD7B interacted with ER $\alpha$ in vitro in a DUB-activitydependent manner. The present study also demonstrated that OTUD7B removed the K11- and K48-linked ubiquitin chain from ER $\alpha$, thus inhibiting proteasome-mediated ER $\alpha$ degradation. In addition, catalytically inactive mutant of OTUD7B (C194S) did not regulate the level of ubiquitination on ER $\alpha$, suggesting that OTUD7B-promoted ER $\alpha$ stability was a consequence of the enzymatically active site of OTUD7B-catalyzed ER $\alpha$ deubiquitination. Our data further demonstrated that OTUD7B depletion dramatically decreased the proliferation and migration of ER $\alpha$-positive breast cancer cells. The suppression effects induced by OTUD7B depletion could be reversed by ER $\alpha$ overexpression. These results demonstrated that OTUD7B promoted breast cancer proliferation and migration through increasing ER $\alpha$ stability.

In the present study, we examined the role of OTUD7B in ER $\alpha$-positive breast cancer cells and identified OTUD7B as the deubiquitinase to mediate ER $\alpha$ deubiquitination. OTUD7B was shown to associate with $E R \alpha$ protein and prolong its stability via removing the K11- and K48-linked ubiquitin chain from ER $\alpha$. Our data suggest that OTUD7B may drive breast tumorigenesis via ER $\alpha$ expression. As ER $\alpha$ signaling plays a central role in ER $\alpha$-positive breast cancer cell proliferation, OTUD7B may be a potential target for breast cancer intervention.

\section{Materials and methods \\ Cell culture}

The human breast cancer cell lines MCF-7, T47D, and human embryonic kidney HEK293 cells were purchased from American Type Culture Collection (ATCC). T47D cells were cultured with RPMI-1640 (42401, Life Technologies) supplemented with $10 \%$ fetal bovine serum (FBS, Gibco, Life Technologies, 10270) and $2 \mathrm{mM} \mathrm{L-glu-}$ tamine (25030, GE Healthcare Hyclone). MCF-7 and HEK293 were cultured with Dulbecco's Modified Eagle's Medium (DMEM) that contains $4 \mathrm{mM}$ L-glutamine and 4,5 g/L glucose (41965, Life Technologies) supplemented 
with $10 \% \mathrm{FBS}$. All cells were cultured at $37^{\circ} \mathrm{C}$ in an atmosphere of $5 \% \mathrm{CO}_{2}$.

\section{Plasmids and RNA inference}

Wild-type (WT) OTUD7B and its inactive mutant plasmids were obtained from Hanbio Biotechnology Co., Ltd. (Shanghai, China). The HA-K6, -K11, -K27, -K29, $-\mathrm{K} 33,-\mathrm{K} 48,-\mathrm{K} 63$, and -Ub plasmids were acquired from Addgene. Small-interfering RNAs targeting OTUD7B (siRNA-1: 5'-CCGAUUGGCCAGUGUAAUU-3'; $5^{\prime}$-CCG AGUGGCUGAUUCCUAU-3') were synthesized by Genepharma (Shanghai, China). The ER $\alpha$ full- and its deletion constructs were gifted from Dr. Ting Zhuang and were described in our previous study ${ }^{30}$. Lipofectamine 2000 (Invitrogen, Carlsbad, CA, USA) was used for plasmid transfection according to the manufacturer's instructions.

\section{RNA extraction and qPCR analysis}

Total RNA was extracted from the cancer cells using the RNeasy plus mini kits (Qiagen, Germany) following the manufacturer's instructions. Reverse transcription was performed using the PrimeScript RT Master Mix (Takara, Japan). qRT-PCR was performed using the SYBR green mix (Toyobo, Japan) with the CFX96TM Real-time PCR Detection System (Bio-Rad, USA) normalized to 36B4. The $2^{-\Delta \Delta \mathrm{Ct}}$ method was used to calculate the relative expression. All assays were performed in triplicates.

\section{Cell proliferation analysis}

The cell proliferation rate was detected using Cell Counting Kit-8 (CCK8) assay at indicated time points according to the manufacturer's instructions. MCF-7 and T47D were transfected with the indicated siRNA, and $24 \mathrm{~h}$ later, cells were digested and $2 \times 10^{3}$ cells were seeded in 96-well culture plates. CCK8 solution reagent was added to each well and incubated for $1.5 \mathrm{~h}$ at $37^{\circ} \mathrm{C}$. The absorbance was measured at $450 \mathrm{~nm}$ using a microplate reader. For clone formation assay, cells were treated with siRNA targeting OTUD7B for $24 \mathrm{~h}$, digested, and seeded into 6-well plates at a density of 1000 cells per well. After 14-day incubation, cells were fixed with $4 \%$ paraformaldehyde and visualized by $0.5 \%$ crystal violet staining. EdU incorporation assay was performed using CellLightTM EdU Apollo 567 In Vitro Kit (Cat number: C10310-1, RiboBio, Guangzhou, China) according to the manufacturer's protocol, and images were captured using an Olympus microscope.

\section{Wound-healing analysis}

MCF-7 and T47D cells were transfected with siRNA targeting OTUD7B. When cells reached full confluency, we scratched the cell layer with a $200-\mu$ sterile pipette tip and washed with PBS. Cells were maintained in the medium containing 1\% FBS and wound distance was measured every $24 \mathrm{~h}$.

\section{Animal experiments}

For xenograft tumor model, female BALB/c nude mice aged 4 weeks (Central Laboratory of Animal Science, Wuhan University, Wuhan, China) were implanted with slow-release 17-beta-estradiol pellets $(0.72 \mathrm{mg} / 90$ days, Innovative Research of America) for one week. Animals were randomly divided into different groups $(n=8$ per group). Stably transfected MCF-7 cells were suspended in PBS $\left(2 \times 10^{6}\right.$ cells $\left./ 100 \mu \mathrm{l}\right)$ and injected into the mammary fat pad. The tumor volume was measured every 4 days until the end of the experiment. For in vivo lung metastasis assays, $2 \times 10^{6}$ MCF-7 cells were injected into female $\mathrm{BALB} / \mathrm{c}$ nude mice via the tail vein. The lung metastasis was examined at the endpoint by routine histopathological analysis. The experiments were approved by the Ethics Committee at Zhongnan Hospital of Wuhan University.

\section{Luciferase assay}

The ERE luciferase reporter plasmid was transfected into MCF-7 cells together with the Renilla plasmid. After $24 \mathrm{~h}$, luciferase activity of ERE luciferase reporter was measured using the dual-Luciferase Reporter kit (Promega, Germany) following the manufacturer's protocol.

\section{Co-immunoprecipitation assay}

Cells were lysed with NP-40 lysis buffer containing a cocktail of protease inhibitors. The total cell lysis was precleared with rabbit IgG for $2 \mathrm{~h}$ and subsequently immunoprecipitated with the indicated antibody at $4{ }^{\circ} \mathrm{C}$ overnight. Protein A/G PLUS-Agarose beads (Santa Cruz) were then added to the lysates and incubated at $4{ }^{\circ} \mathrm{C}$ for $2 \mathrm{~h}$. The immunocomplexes were washed with lysis buffer three times and separated by SDS-PAGE. Immunoblotting was performed following standard procedures.

\section{GST pull-down assays}

Bacterial-expressed GST and GST-ER $\alpha$ bound to glutathione-Sepharose 4B beads (from GE) was incubated with recombinant His-OTUD7B at $4{ }^{\circ} \mathrm{C}$ for $2 \mathrm{~h}$. Then the beads were washed with GST-binding buffer. The bound proteins were separated by SDS-PAGE, followed by Western blot with indicated antibodies.

\section{In vivo deubiquitination assay}

In vivo deubiquitination assay was performed in HEK293 and breast cancer cells. HEK293 cells were transfected with HA-Ub, Flag-ER $\alpha$, Myc-OTUD7B, or Myc-OTUD7B ${ }^{\text {C194S }}$ plasmid as indicated for $48 \mathrm{~h}$. After 6-h incubation with $10 \mu \mathrm{M}$ MG132 (MCE), cells were harvested and immunoprecipitated to isolate HA-ubiquitinated ER $\alpha$. ER $\alpha$ ubiquitination was detected by Western blotting with the 
indicated antibodies. In breast cancer cells, HA-Ub plasmid was cotransfected with OTUD7B siRNAs into MCF-7 cells. After $24 \mathrm{~h}$, cells were treated with $10 \mu \mathrm{M}$ proteasome inhibitor MG132 for $6 \mathrm{~h}$, harvested, and incubated with anti-ER $\alpha$ antibody (CST) for $2 \mathrm{~h}$ and protein $\mathrm{A} / \mathrm{G}$ agarose beads (Santa Cruz) overnight at $4{ }^{\circ} \mathrm{C}$. The immunocomplexes were washed with lysis buffer three times and ER $\alpha$ ubiquitination was detected by Western blotting.

\section{Western blot analysis}

Cells were lysed with RIPA extraction reagent (Beyotime, China) supplemented with protease inhibitors (Sigma-Aldrich, USA). Total protein was separated using $10-12.5 \%$ sodium dodecyl sulfate polyacrylamide gel electrophoresis and transferred to 0.45- $\mu \mathrm{m}$ PVDF membrane (Millipore, USA). Primary antibodies were ER $\alpha$ (CST, 8644), OTUD7B (Proteintech, 66276-1-Ig), HA (Proteintech, 51064-2-AP), Myc (Proteintech, 60003-2Ig), and GAPDH (Proteintech, 60004-1-Ig) antibodies. Bands were visualized using an enhanced chemiluminescence (ECL) kit (Boster, China) and detected by ChemiDoc XRS + Imaging System (Bio-Rad).

\section{Statistical analysis}

Student's $t$ test and one-way ANOVA were used to compare two and more groups respectively. Multiple comparison with Bonferroni correction was performed when appropriate. A $P$ value $<0.05$ was considered as statistically significant and all tests were two-tailed. All statistical tests were performed with Prism 7.0 (GraphPad, USA).

\section{Acknowledgements}

We thank Pro. Ting Zhuang and Jian Zhu (Henan Key Laboratory of immunology and targeted therapy, Xinxiang Medical University) for kindly providing ERa full-/ deletion constructs plasmids and estrogen-response-element (ERE)-TK reporter plasmids. This work was supported by the Improvement Project for Theranostic ability on Difficulty miscellaneous disease (Tumor).

\section{Author contributions}

J.T. performed most of the benchwork. Z.T., Z.W., and W.C. participated in the modification and prognosis analysis of the paper. G.W. supervised the process of the study and wrote the paper. All authors have read and approved the final paper.

\section{Ethics statement}

The research was carried out according to the World Medical Association Declaration of Helsinki and was approved by the Ethics Committee at Zhongnan Hospital of Wuhan University.

\section{Funding}

This work was supported by the Improvement Project for Theranostic ability on Difficulty miscellaneous disease (Tumor).

\section{Conflict of interest}

The authors declare no competing interests.

\section{Publisher's note}

Springer Nature remains neutral with regard to jurisdictional claims in published maps and institutional affiliations.
Supplementary information The online version contains supplementary material available at https://doi.org/10.1038/s41419-021-03785-7 .

Received: 22 September 2020 Revised: 2 February 2021 Accepted: 5 February 2021

Published online: 25 May 2021

\section{References}

1. Bray, F. et al. Global cancer statistics 2018: GLOBOCAN estimates of incidence and mortality worldwide for 36 cancers in 185 countries. CA Cancer J. Clin. $6 \mathbf{8}$, 394-424 (2018)

2. Waks, A. G. \& Winer, E. P. Breast cancer treatment: a review. JAMA 321, 288-300 (2019).

3. Sledge, G. W. et al. Past, present, and future challenges in breast cancer treatment. J. Clin. Oncol. 32, 1979-1986 (2014).

4. Nicolini, A., Ferrari, P. \& Duffy, M. J. Prognostic and predictive biomarkers in breast cancer: past, present and future. Semin. Cancer Biol. 52, 56-73 (2018).

5. Ng, H. W., Perkins, R., Tong, W. \& Hong, H. Versatility or promiscuity: the estrogen receptors, control of ligand selectivity and an update on subtype selective ligands. Int. J. Environ. Res. Public Health 11, $8709-8742$ (2014).

6. Greene, G. L. \& Press, M. F. Structure and dynamics of the estrogen receptor. J. Steroid Biochem. 24, 1-7 (1986)

7. Miyoshi, Y., Murase, K., Saito, M., Imamura, M. \& Oh, K. Mechanisms of estrogen receptor-a upregulation in breast cancers. Med. Mol. Morphol. 43, 193-196 (2010).

8. Tecalco-Cruz, A. C. \& Ramírez-Jarquín, J. O. Mechanisms that increase stability of estrogen receptor alpha in breast cancer. Clin. Breast Cancer 17, 1-10 (2017).

9. Giulianelli, S. et al. [The role of estrogen receptor alpha in breast cancer cell proliferation mediated by progestins]. Medicina 72, 315-320 (2012).

10. Tecalco-Cruz, A. C., Ramirez-Jarquin, J. O. \& Cruz-Ramos, E. Estrogen receptor alpha and its ubiquitination in breast cancer cells. Curr. Drug Targets 20, 690-704 (2019).

11. Ignatiadis, M. \& Sotiriou, C. Luminal breast cancer: from biology to treatment. Nat. Rev. Clin. Oncol. 10, 494-506 (2013).

12. Matsumoto, A. et al. Prognostic implications of receptor discordance between primary and recurrent breast cancer. Int. J. Clin. Oncol. 20, 701-708 (2015).

13. Zhang, X. H., Giuliano, M., Trivedi, M. V., Schiff, R. \& Osborne, C. K. Metastasis dormancy in estrogen receptor-positive breast cancer. Clin. Cancer Res. 19, 6389-6397 (2013).

14. Johnson, A. B. \& O'Malley, B. W. Erasing breast cancer resistance through the kinome. Nat. Med. 17, 660-661 (2011).

15. Abdel-Hafi, A. et al. Epigenetic mechanisms of tamoxifen resistance in luminal breast cancer. Diseases 5, 16 (2017)

16. Abdel-Hafiz, H. A. \& Horwitz, K. B. Role of epigenetic modifications in luminal breast cancer. Epigenomics 7, 847-862 (2015).

17. Zhang, J. et al. Mechanisms of resistance to estrogen receptor modulators in ER+/HER2- advanced breast cancer. Cell. Mol. Life Sci. 77, 559-572 (2020).

18. Donley, C. et al. Identification of RBCK1 as a novel regulator of FKBPL: implications for tumor growth and response to tamoxifen. Oncogene 33 3441-3450 (2014).

19. Karamouzis, M. V., Konstantinopoulos, P. A., Badra, F. A. \& Papavassiliou, A. G. SUMO and estrogen receptors in breast cancer. Breast Cancer Res. Treat. 107, 195-210 (2008)

20. Zhuang, T. et al. p21-activated kinase group II small compound inhibitor GNE2861 perturbs estrogen receptor alpha signaling and restores tamoxifensensitivity in breast cancer cells. Oncotarget 6, 43853-43868 (2015).

21. Fan, M., Park, A. \& Nephew, K. P. CHIP (carboxyl terminus of Hsc70-interacting protein) promotes basal and geldanamycin-induced degradation of estrogen receptor-alpha. Mol. Endocrinol. 19, 2901-2914 (2005).

22. Sun, J., Zhou, W., Kaliappan, K., Nawaz, Z. \& Slingerland, J. M. ERa phosphorylation at Y537 by Src triggers E6-AP-ERa binding, ERa ubiquitylation, promoter occupancy, and target gene expression. Mol. Endocrinol. 26, 1567-1577 (2012).

23. Eakin, C. M., Maccoss, M. J., Finney, G. L. \& Klevit, R. E. Estrogen receptor alpha is a putative substrate for the BRCA1 ubiquitin ligase. Proc. Natl Acad. Sci. USA 104, 5794-5799 (2007).

24. Hashizume, R. et al. The RING heterodimer BRCA1-BARD1 is a ubiquitin ligase inactivated by a breast cancer-derived mutation. J. Biol. Chem. 276 14537-14540 (2001) 
25. Bhatt, S., Xiao, Z., Meng, Z. \& Katzenellenbogen, B. S. Phosphorylation by p38 mitogen-activated protein kinase promotes estrogen receptor a turnover and functional activity via the SCF(Skp2) proteasomal complex. Mol. Cell. Biol. 32 1928-1943 (2012)

26. Saji, S. et al. MDM2 enhances the function of estrogen receptor alpha in human breast cancer cells. Biochem. Biophys. Res. Commun. 281 259-265 (2001).

27. Zhuang, T. et al. SHARPIN stabilizes estrogen receptor alpha and promotes breast cancer cell proliferation. Oncotarget 8, 77137-77151 (2017).

28. Zhu, J. et al. The atypical ubiquitin ligase RNF31 stabilizes estrogen receptor alpha and modulates estrogen-stimulated breast cancer cell proliferation. Oncogene 33, 4340-4351 (2014).

29. Wang, S. et al. RNF8 identified as a co-activator of estrogen receptor alpha promotes cell growth in breast cancer. Biochim. Biophys. Acta Mol. Basis Dis. 1863, 1615-1628 (2017).

30. Tang, J. et al. TRIM11 promotes breast cancer cell proliferation by stabilizing estrogen receptor a. Neoplasia 22, 343-351 (2020).

31. Xue, M. et al. Regulation of estrogen signaling and breast cancer proliferation by an ubiquitin ligase TRIM56. Oncogenesis 8, 30 (2019).

32. Xia, $\mathrm{X}$. et al. Deubiquitination and stabilization of estrogen receptor a by ubiquitin-specific protease 7 promotes breast tumorigenesis. Cancer Lett. $\mathbf{4 6 5}$, 118-128 (2019).

33. Tian, Z. et al. TRIM8 inhibits breast cancer proliferation by regulating estrogen signaling. Am. J. Cancer Res. 10, 3440-3457 (2020).

34. Onitilo, A. A., Engel, J. M., Greenlee, R. T. \& Mukesh, B. N. Breast cancer subtypes based on ER/PR and Her2 expression: comparison of clinicopathologic features and survival. Clin. Med. Res. 7, 4-13 (2009).

35. Walter, P. et al. Cloning of the human estrogen receptor cDNA. Proc. Natl Acad. Sci. USA 82, 7889-7893 (1985).

36. Shao, W. \& Brown, M. Advances in estrogen receptor biology: prospects for improvements in targeted breast cancer therapy. Breast Cancer Res. 6, 39-52 (2004).
37. Karnik, P. S., Kulkarni, S., Liu, X. P., Budd, G. T. \& Bukowski, R. M. Estrogen receptor mutations in tamoxifen-resistant breast cancer. Cancer Res. 54 349-353 (1994).

38. Wang, C. et al. Direct acetylation of the estrogen receptor alpha hinge region by p300 regulates transactivation and hormone sensitivity. J. Biol. Chem. $\mathbf{2 7 6}$ 18375-18383 (2001).

39. Anbalagan, M. \& Rowan, B. G. Estrogen receptor alpha phosphorylation and its functional impact in human breast cancer. Mol. Cell. Endocrinol. 418, 264-272 (2015).

40. Tharun, I. M. et al. Subtype-specific modulation of estrogen receptorcoactivator interaction by phosphorylation. ACS Chem. Biol. 10, 475-484 (2015).

41. Bonacci, T. et al. Cezanne/OTUD7B is a cell cycle-regulated deubiquitinase that antagonizes the degradation of APC/C substrates. EMBO J. 37, e98701 (2018).

42. Bremm, A., Freund, S. M. \& Komander, D. Lys11-linked ubiquitin chains adopt compact conformations and are preferentially hydrolyzed by the deubiquitinase Cezanne. Nat. Struct. Mol. Biol. 17, 939-947 (2010)

43. Mevissen, T. E. et al. OTU deubiquitinases reveal mechanisms of linkage specificity and enable ubiquitin chain restriction analysis. Cell 154, 169-184 (2013).

44. Mevissen, T. E. T. et al. Molecular basis of Lys11-polyubiquitin specificity in the deubiquitinase Cezanne. Nature 538, 402-405 (2016).

45. $\mathrm{Hu}, \mathrm{H}$. et al. OTUD7B controls non-canonical NF-kB activation through deubiquitination of TRAF3. Nature 494, 371-374 (2013).

46. Pareja, F. et al. Deubiquitination of EGFR by Cezanne-1 contributes to cancer progression. Oncogene 31, 4599-4608 (2012).

47. Bremm, A., Moniz, S., Mader, J., Rocha, S. \& Komander, D. Cezanne (OTUD7B) regulates HIF-1a homeostasis in a proteasome-independent manner. EMBO Rep. 15, 1268-1277 (2014).

48. Cui, C. P. et al. Dynamic ubiquitylation of Sox2 regulates proteostasis and governs neural progenitor cell differentiation. Nat. Commun. 9, 4648 (2018). 